\title{
Alzheimer's Disease Neuropathological Comorbidities Are Common in the Younger-Old
}

\section{Thomas G. Beach ${ }^{1}$ and Michael Malek-Ahmadi ${ }^{2}$}

1. Banner Sun Health Research Institute, Sun City, Arizona

2. Banner Alzheimer's Institute, Phoenix, Arizona 


\section{Abstract}

Clinicopathological studies have demonstrated that Alzheimer's disease dementia (ADD) is often accompanied by clinically undetectable comorbid neurodegenerative and cerebrovascular disease that alter the presence and rate of cognitive decline in aging and ADD. Aside from causing increased variability in clinical response, it is possible that the major ADD comorbidities may not respond to ADD-specific molecular therapeutics. As most reports have focused on comorbidity in the oldest-old, its extent in younger age groups that are more likely to be involved in clinical trials is largely unknown. We conducted a survey of neuropathological comorbidities in sporadic ADD using data from the US National Alzheimer's Coordinating Center. Subject data was restricted to those with dementia and meeting National Institute on AgingAlzheimer's Association (NIA-AA) intermediate or high AD Neuropathological Change (ADNC) levels, excluding those with known autosomal dominant AD-related mutations. Subjects were divided into age-atdeath categories for analysis: under $60,60-69,70-79,80-89,90-99$ and 100 or over. Confirmatory of earlier reports, ADD histopathology is less severe with advancing age, effectively increasing the relative contribution of comorbidities, most of which rise in prevalence with age. Highly prevalent ADD comorbidities are not restricted to the oldest-old but are common even in early-onset ADD. The percentage of cases with ADD as the sole major neuropathological diagnosis is highest in the under-60 group, where "pure" ADD cases are still in the minority at $44 \%$. After this AD as a sole major pathology in ADD declines to roughly $20 \%$ in the 70 s and beyond. Comorbidity rates for some pathologies, especially LBD, are high even in subjects in their 60s and 70 s, at nearly $60 \%$, but for most others, their prevalence increases with age. TDP-43 pathology affects more than $35 \%$ of ADD subjects 80 and over while microscopic infarcts reach this rate a decade later. Gross infarcts rise more slowly and affect fewer subjects but still involve $15-20 \%$ of ADD after age 80 . White matter rarefaction may be underestimated in the NACC database but is present in almost $70 \%$ of centenarians with ADD. Effective clinical trials depend on accurate estimates of required subject numbers, which are dependent on observed effect size and clinical response variability. Comorbidities are likely to affect both, leading to lower probability of clinical trial success. Stratifying ADD clinical trial analyses by presence and types of accompanying comorbidities might identify subgroups with higher effect sizes and greater clinical response rates, but accurate in-vivo diagnostic methods for most comorbidities are still lacking. 


\section{Introduction}

Neuropathological studies are increasingly demonstrating that Alzheimer's disease dementia (ADD) is most often not the sole brain pathology in those that die and are autopsied, but is very likely to be accompanied by comorbid neurodegenerative and cerebrovascular disease. Comorbidities are likely to alter the presence and rate of cognitive decline in aging and ADD [1-40]. If all neuropathological changes found in elderly brains were considered, there would be few if any cases of "pure" ADD. While not all of these aging changes are likely be clinically significant [37], several of the commonly-observed ADD comorbidities have been demonstrated to contribute to cognitive impairment, including Lewy body disease (LBD) [4,23,26,30,41-47], hippocampal sclerosis [29,47-52], TDP-43 proteinopathy [12,13,37,53-57] and cerebral infarcts $[1,10,11,30,35,46,57,58,58-$ 70]. Aside from causing increased variability in clinical response, it is possible that these major comorbidities, each of which is individually capable of causing dementia, may not respond to ADD-specific molecular therapeutics because their molecular pathogenesis is different from ADD. At present, there are no accurate methods available with which to clinically diagnose these complications of ADD.

As the reported studies of comorbidities have focused on the oldest-old, and an increase in their prevalences with increasing age has been noted [19], it has been assumed by some that these would largely not be present in younger aged ADD subjects that might be more likely to be involved in clinical trials. However, in the Arizona Study of Aging and Neurodegenerative Disorders/Banner Sun Health Research Institute Brain and Body Donation Program [71], although the number of comorbid conditions increased with each decade, even subjects in their 70s had, on average, 2.8 major neuropathological diagnoses at autopsy [27]. To determine whether these findings were more broadly confirmed, we conducted a survey of neuropathological comorbidities in sporadic ADD using data from the National Alzheimer's Coordinating Center (NACC) [72,73].

\section{Methods}

We used a recent (10/09/19) NACC data download of all clinical and autopsy data with entries on NACC NP form version 10 [73]. The time period covered therefore is from January 2014 through August 2019. Included subject data was restricted to those with dementia and meeting National Institute on AgingAlzheimer's Association (NIA-AA) intermediate or high AD Neuropathological Change (ADNC) levels [74,75], considered to be a sufficient cause of cognitive impairment or dementia. Subjects known to have autosomal dominant AD-related mutations were excluded. Subjects were divided into age-at-death categories for analysis: under 60, 60-69, 70-79, 80-89, 90-99 and 100 or over.

Pathology categories investigated consisted of the major AD-specific lesions including senile plaques of all types, classified by Thal amyloid phase [76] (NACC variable NPTHAL), neurofibrillary tangles, classified by Braak stage stage [77,78] (NACC variable NPBRAAK), neuritic plaques, classified according to CERAD [79] (NACC variable NPNEUR), diffuse plaques, classified analogously to CERAD neuritic plaques (NACC variable NPDIFF), amyloid angiopathy, classified as none, mild, moderate or severe (NPAMY), and NIA-AA AD Neuropathological Change (ADNC) Level, with categories of intermediate or high [74,75](NACC variable NPADNC). Means and medians for each age category were compared. Decadal groups were compared using Kruskal-Wallis analysis of variance for ordinal AD pathology measures and chi-square tests were used to compare comorbidity prevalence rates.

Comorbid neurodegenerative conditions investigated included Lewy body disease (NACC variable NPLBOD; for this study only presence or absence in any brain region was recorded), hippocampal sclerosis (NACC variable NPHIPSCL; for this study recorded as present or absent); TDP-43 pathology (NACC variable NPTDPB; TDP-43 pathology present in amygdala), non-AD tauopathy (NACC variable NPFTDTAU; any nonAD tauopathy including progressive supranuclear palsy, corticobasal degeneration, Pick's disease, argyrophilic grains, chronic traumatic encephalopathy, "tangle-only disease" or "other"). For each comorbid pathology and age category, the proportion of cases possessing that pathology was determined, relative to the number of cases for which the specific pathology type was assessed by contributing neuropathologists.

Comorbid cerebrovascular conditions investigated included circle of Willis arteriosclerosis (NACC variable NACCAVAS; for this study only "moderate" and "severe" qualified for presence of the condition), old gross cerebral infarcts (NACC variable NPINF; for this study 1 or more large or lacunar infarcts qualified for the condition), old microscopic infarcts (NACC variable NPOLD; for this study 1 or more old microinfarcts qualified 
for the condition), old microscopic hemorrhages (NACC variable NPOLDD; for this study 1 or more old microhemorrhages qualified for the condition), arteriolosclerosis (NACC variable NACCARTE; for this study only "moderate" and "severe" qualified for presence of the condition) and white matter rarefaction (NACC variable NPWMR; for this study only "moderate" and "severe" qualified for presence of the condition). For each comorbid pathology and age category, the proportion of cases possessing that pathology, relative to those for whom the pathology's presence or absence was specifically recorded, was determined.

For all conditions, cases with missing data, or for which the specific condition was not assessed, were excluded.

\section{Results and Discussion}

There were 1,839 cases that had dementia and met NIA-AA intermediate or high ADNC levels (Table 1). Confirmatory of earlier reports [19,52,80,81], ADD histopathology consistently becomes less severe with age (Table 1). It is important to realize that the decreased severity of AD histopathological lesions with age would effectively increase the relative importance of comorbidities in older subjects, most of which rise in prevalence with age.

Our analysis of these data shows that highly prevalent ADD comorbidities are not restricted to the oldestold but are common even in early-onset ADD. Comorbidity rates for some pathologies, especially LBD, are high even in subjects in their 60s and 70s (Tables 2 and 3, Figure 1). The percentage of cases with ADD as the sole major neuropathological diagnosis, defined as the absence of LBD, TDP-43 pathology, non-AD tauopathy, hippocampal sclerosis, infarcts and microhemorrrhages, is highest in the under-60 group, where "pure" ADD cases are still in the minority, at $44 \%$. After this AD as a sole major pathology in ADD declines to roughly $20 \%$ in the 70 s and beyond.

Lewy body disease is the most common ADD comorbidity in subjects under 80 , and in fact is most prevalent in $A D$ subjects under 60, with its proportional presence gradually declining in older decades (Figure 1). As this closely parallels the decline in AD pathology severity with age, one conclusion is that LBD is largely synchronous or even dependent on AD pathology. The high reported rate of Lewy body disease in autosomal dominant ADD [82-89], and even in other cerebral amyloidoses [90,91] supports this conclusion. Prior work has consistently found that one-half or more of all subjects meeting clinicopathological diagnostic criteria for ADD also have LBD [71,92-94]. Similarly, up to one-half of subjects with dementia and Parkinson's disease [95-107] and three-quarters or more of those with DLB, have clinically significant AD pathology [108-111]. In the great majority of subjects with both ADD and LBD, this co-existence is recognized only at autopsy [41,112$115]$, currently preventing, except for the minority with clinically-typical DLB, the exclusion or stratification of LBD subjects within ADD clinical trials.

The presence of LBD in ADD is clearly of clinical significance. Multiple autopsy-validated studies have indicated that cognitive decline is faster in ADD with any degree of associated LBD [26,42-45]. Formerly, it was not apparent whether or not this was primarily driven by dementia with Lewy bodies (DLB) subjects with neuropathologically-severe LBD (mostly neocortical stage diffuse Lewy body disease), as disease duration is reportedly shorter in this group [109,116]. The great majority of LBD in ADD subjects, however, does not meet neuropathological diagnostic criteria for DLB, due to insufficient pathology density and brain regional distribution [117-119]. These "AD-LB" cases are most often clinically silent [24] and diagnosed as probable ADD. We have recently reported that even in these AD-LB subjects without neocortical disease, as compared to ADD subjects without any LBD, that depression and Trail-Making Test A scores correlate significantly with LBD pathology, and the global rate of cognitive decline is more rapid than in either AD-DLB or ADD without LBD [41,120], even after adjustment for AD pathology severity. These data suggest that accompanying LBD is a "state" biomarker of a more clinically severe form of AD.

All the other ADD comorbidities increase in prevalence with age, suggesting that they are primarily agedependent, or at least co-dependent on both AD and age (Figure 1). Of these, hippocampal sclerosis and TDP-43 pathology, which are thought to be closely related or even subsets of a single pathogenic class [50,80] are the next most common ADD comorbidity, at least up to about age 80-90, when TDP-43 pathology is present in about $35 \%$ of ADD cases (Table 2, Figure 1). Comorbid TDP-43 has been reported by several groups to contribute to cognitive impairment in ADD [12,13,37,53-57]. 
Cerebrovascular disease, as a whole, may be of equal or greater importance as an ADD comorbidity, as compared to LBD and TDP-43. Microscopic infarcts rise steadily in prevalence with age, at 40-50\% becoming the most common ADD comorbidity after age 90. Gross infarcts rise more slowly and affect fewer subjects but still involve $15-20 \%$ of ADD after age 80 . Both of these infarct classes have been reported to be independent influences on cognition [1,10,11,30,35,57-69]. White matter rarefaction (WMR) has long been reported to be roughly twice as common in ADD as compared to normal elderly, being present in $60 \%$ or more of ADD subjects [121-123]. At 30-70\%, the NACC database may be underestimating WMR. This may be due to the difficulty of detecting it with only standard small-block histology sampling. Without setting thresholds of severity, MRI-detected WMR has been reported to be present in 39-96\% of elderly subjects and close to $100 \%$ of those with ADD [124]. Correlations of WMR with cognition have been complicated by co-linearity with AD histopathology as well as a dual vascular and AD-related pathogenesis [58,70,125-132], but when WMR rarefaction in ADD is severe, or in non-demented older subjects where AD histopathology is minimal, there are significant associations, especially with frontal lobe functions or longitudinal decline [124,133-136]. Like LBD, WMR is highly prevalent in autosomal dominant early-onset AD and thus is probably at least partially dependent on AD-related factors [137-139].

In the NACC database, microhemorrhages are much less common than infarcts and WMR, reaching only $5-8 \%$ prevalence after age 80 . Again these may be underestimated at autopsy as compared to MRI, or when special efforts are made in postmortem examination, as one autopsy center has reported a prevalence rate of $62 \%$ in oldest-old subjects [140]. In the Alzheimer's Disease Neuroimaging Initiative, $25 \%$ of a mixed group of subjects, with normal cognition, mild cognitive impairment, and ADD, had microhemorrhages, with a tendency for proportionately more in AD [141]. Microhemorrhages are reported to increase risk for incident dementia [142].

Non-AD tauopathies, with microscopic tau pathologist that is morphologically unlike the neurofibrillary tangles of $A D$, are relatively uncommon ADD comorbidities in the NACC database, being present in under $10 \%$ or less of subjects under 90 . It is possible that these tauopathies may often be overlooked against the background of severe AD neurofibrillary pathology. Reports from centers that specifically look for argyrophilic grains and aging-related tau astrogliopathy (ARTAG) find AD comorbidity rates up to $40 \%$ for both [143-150]. The classical conditions in this group, including progressive supranuclear palsy (PSP), corticobasal degeneration (CBD) and Pick's disease, are much less prevalent, although PSP may be considerably more common than previously realized due to low clinical sensitivity for the diagnosis [143,151-158].

Effective clinical trials depend on accurate estimates of required subject numbers and variability as well as effect size of the treatment. Increased variability of clinical decline rates will require larger subject numbers to overcome. Effect size depends on the efficacy of the therapeutic agent, which may be at least in part dependent on a matching of molecular mechanisms of agent and pathogenesis. Neuropathological comorbidities in AD affect rates of cognitive decline and are by definition different in their molecular pathogenesis from AD. Comorbidities are thus likely to lead to lower probability of clinical trial success. Stratifying AD subjects by presence and types of accompanying comorbidities might result in increased observed effect sizes in some groups as compared to others, potentially "rescuing" failed clinical trials. Accurate in-vivo diagnostic methods are urgently needed for the major AD comorbidities.

\section{Acknowledgements}

The NACC database is funded by NIA/NIH Grant U01 AG016976. NACC data are contributed by the NIA-funded ADCs: P30 AG019610 (PI Eric Reiman, MD), P30 AG013846 (PI Neil Kowall, MD), P30 AG062428-01 (PI James Leverenz, MD) P50 AG008702 (PI Scott Small, MD), P50 AG025688 (PI Allan Levey, MD, PhD), P50 AG047266 (PI Todd Golde, MD, PhD), P30 AG010133 (PI Andrew Saykin, PsyD), P50 AG005146 (PI Marilyn Albert, PhD), P30 AG062421-01 (PI Bradley Hyman, MD, PhD), P30 AG062422-01 (PI Ronald Petersen, MD, PhD), P50 AG005138 (PI Mary Sano, PhD), P30 AG008051 (PI Thomas Wisniewski, MD), P30 AG013854 (PI Robert Vassar, PhD), P30 AG008017 (PI Jeffrey Kaye, MD), P30 AG010161 (PI David Bennett, MD), P50 AG047366 (PI Victor Henderson, MD, MS), P30 AG010129 (PI Charles DeCarli, MD), P50 AG016573 (PI Frank LaFerla, PhD), P30 AG062429-01(PI James Brewer, MD, PhD), P50 AG023501 (PI Bruce Miller, MD), P30 AG035982 (PI Russell Swerdlow, MD), P30 AG028383 (PI Linda Van Eldik, PhD), P30 AG053760 (PI Henry Paulson, MD, PhD), P30 AG010124 (PI John Trojanowski, MD, PhD), P50 AG005133 (PI Oscar Lopez, MD), P50 AG005142 (PI Helena Chui, MD), P30 AG012300 (PI Roger Rosenberg, MD), P30 AG049638 (PI Suzanne Craft, PhD), P50 AG005136 (PI Thomas Grabowski, MD), P30 AG062715-01 (PI Sanjay Asthana, MD, FRCP), P50 AG005681 (PI John Morris, MD), P50 AG047270 (PI Stephen Strittmatter, MD, PhD). 
Table 1. NACC autopsy data, by decade, for basic AD-related neuropathological variables in 1,839 dementia cases with intermediate or high ADNC. Number of cases, means and medians are shown. NPTHAL $=$ Thal amyloid phase; NPBRAAK = Braak neurofibrillary stage; NPNEUR = CERAD neuritic plaque density; NPDIFF = diffuse plaque density; NPAMY = cerebral amyloid angiopathy density; NPADNC = NIA-AA AD

Neuropathological Change level. Decadal groups are significantly different $(p<0.000001$ for all $A D$ lesion types except NPDIFF $(p=0.00046)$ and NPAMY $(p=0.0126)$.

\begin{tabular}{|l|c|c|c|c|c|c|}
\hline \multicolumn{1}{|c|}{ Age } & NPTHAL & NPBRAAK & NPNEUR & NPDIFF & NPAMY & NPADNC \\
\hline$<60(n=33)$ & $4.72 ; 5$ & $5.79 ; 6$ & $2.85 ; 3$ & $2.70 ; 3$ & $1.42 ; 1$ & $2.88 ; 3$ \\
\hline $60-69(n=230)$ & $4.71 ; 5$ & $5.72 ; 6$ & $2.86 ; 3$ & $2.73 ; 3$ & $1.45 ; 1$ & $2.90 ; 3$ \\
\hline $70-79(n=436)$ & $4.56 ; 5$ & $5.47 ; 6$ & $2.81 ; 3$ & $2.80 ; 3$ & $1.49 ; 1$ & $2.82 ; 3$ \\
\hline $80-89(n=624)$ & $4.42 ; 5$ & $5.23 ; 6$ & $2.63 ; 3$ & $2.76 ; 3$ & $1.38 ; 1$ & $2.73 ; 3$ \\
\hline $90-99(n=385)$ & $4.10 ; 4$ & $4.87 ; 5$ & $2.32 ; 2$ & $2.66 ; 3$ & $1.24 ; 1$ & $2.54 ; 3$ \\
\hline$>99(n=38)$ & $4.10 ; 4$ & $4.66 ; 4$ & $2.18 ; 2$ & $2.55 ; 3$ & $1.50 ; 1$ & $2.34 ; 2$ \\
\hline
\end{tabular}

Table 2. NACC data, by decade, for proportions and percentages of common neurodegenerative AD comorbidities in dementia cases with intermediate or high NIA-AA ADNC. Numerator is the number of cases meeting criteria for the comorbidity while denominator is the number of AD cases evaluated for the condition. $A D$ Only = AD without any of the other diagnoses in this table and without NPINF, NPOLD and NPOLDD from Table 3; NPLBOD = Lewy body disease; NPHIPSCL = hippocampal sclerosis; TDPNOS = TDP-43 pathology at minimum present in amygdala; NPFTDTAU = non-AD tau pathology (PSP, CBD, Pick's, argyrophilic grains, other). Decadal groups are significantly different $(p<0.001)$ except for AD Only $(p=0.02)$.

\begin{tabular}{|c|c|c|c|c|c|}
\hline Age & $\begin{array}{c}\text { AD Only } \\
(\mathbf{n = 8 3 5})\end{array}$ & $\begin{array}{c}\text { NPLBOD } \\
(\mathbf{n = 1}, \mathbf{7 3 7})\end{array}$ & $\begin{array}{c}\text { NPHIPSCL } \\
(\mathbf{n = 1}, \mathbf{8 0 0})\end{array}$ & $\begin{array}{c}\text { TDPNOS } \\
(\mathbf{n = 1 , 0 0 6})\end{array}$ & $\begin{array}{c}\text { NPFTDTAU } \\
(\mathbf{n = 1 , 8 1 2})\end{array}$ \\
\hline$<60$ & $8 / 18 ; 44.4 \%$ & $19 / 33 ; 57.6 \%$ & $1 / 32 ; 3.1 \%$ & $2 / 18 ; 11.1 \%$ & $1 / 33 ; 3.0 \%$ \\
\hline $60-69$ & $40 / 119 ; 33.6 \%$ & $111 / 229 ; 48.5 \%$ & $19 / 320 ; 5.9 \%$ & $9 / 229 ; 3.9 \%$ & $10 / 320 ; 0.3 \%$ \\
\hline $70-79$ & $52 / 226 ; 23.0 \%$ & $221 / 432 ; 51.2 \%$ & $46 / 424 ; 10.8 \%$ & $66 / 251 ; 26.3 \%$ & $35 / 429 ; 8.2 \%$ \\
\hline $80-89$ & $63 / 308 ; 20.4 \%$ & $288 / 622 ; 46.3 \% ;$ & $113 / 610 ; 18.5 \%$ & $122 / 339 ; 36.0 \%$ & $56 / 617 ; 9.1 \%$ \\
\hline $90-99$ & $33 / 149 ; 22.1 \%$ & $119 / 383 ; 31.1 \%$ & $56 / 376 ; 14.9 \%$ & $54 / 154 ; 35.1 \%$ & $40 / 375 ; 10.7 \%$ \\
\hline$>99$ & $3 / 15 ; 20 \%$ & $12 / 38 ; 31.6 \%$ & $8 / 38 ; 21.0 \%$ & $6 / 15 ; 40 \%$ & $8 / 38 ; 21.0 \%$ \\
\hline
\end{tabular}

Table 3. NACC data, by decade, for proportions and percentages of common AD cerebrovascular comorbidities in dementia cases with intermediate or high NIA-AA ADNC. Numerator is the number of cases meeting criteria for the condition, denominator is the number of cases evaluated for the condition. NACCAVAS $=$ severity of atherosclerosis of circle of Willis $>$ mild; NPINF = gross infarcts including lacunes; NPOLD $=$ old microinfarcts; NPOLDD = old cerebral microhemorrhages; NACCARTE $=$ arteriolosclerosis $>$ mild; NPWMR $=$ white matter rarefaction $>$ mild. Decadal groups are significantly different $(p<0.001)$ except for NPOLDD $(p=$ 0.05).

\begin{tabular}{|c|c|c|c|c|c|c|}
\hline Age & $\begin{array}{c}\text { NACCAVAS } \\
(\mathbf{n = 1 , 7 0 7 )}\end{array}$ & $\begin{array}{c}\text { NPINF } \\
(\mathbf{n = 1 , 8 3 2})\end{array}$ & $\begin{array}{c}\text { NPOLD } \\
(\mathbf{n}=\mathbf{1 , 8 3 4})\end{array}$ & $\begin{array}{c}\text { NPOLDD } \\
(\mathbf{n}=\mathbf{1 , 7 6 5 )}\end{array}$ & $\begin{array}{c}\text { NACCARTE } \\
(\mathbf{n}=\mathbf{1 , 7 1 5})\end{array}$ & $\begin{array}{c}\text { NPWMR } \\
(\mathbf{n = 1 , 6 5 7 )}\end{array}$ \\
\hline$<60$ & $1 / 33 ; 3.0 \%$ & $0 / 33 ; 0 \%$ & $2 / 33 ; 6.1 \%$ & $0 / 29 ; 0 \%$ & $6 / 32 ; 18.7 \%$ & $5 / 28 ; 17.9 \%$ \\
\hline $60-69$ & $39 / 319 ; 12.2 \%$ & $10 / 321 ; 3.1 \%$ & $25 / 322 ; 7.8 \%$ & $9 / 307 ; 2.7 \%$ & $77 / 309 ; 24.9 \%$ & $57 / 296 / 19.3 \%$ \\
\hline $70-79$ & $145 / 317 ; 45.7 \%$ & $48 / 435 ; 11.0 \%$ & $84 / 435 ; 19.3 \%$ & $24 / 411 ; 5.8 \%$ & $176 / 413 ; 42.6 \%$ & $111 / 379 ; 33.7 \%$ \\
\hline $80-89$ & $275 / 616 ; 44.6 \%$ & $102 / 621 ; 16.4 \%$ & $154 / 622 ; 24.8 \%$ & $16 / 600 ; 2.7 \%$ & $326 / 577 ; 56.5 \%$ & $166 / 556 ; 29.9 \%$ \\
\hline $90-99$ & $225 / 384 ; 58.6 \%$ & $79 / 384 ; 20.6 \%$ & $147 / 384 ; 38.3 \%$ & $19 / 380 ; 5.0 \%$ & $209 / 352 ; 59.4 \%$ & $104 / 360 ; 28.9 \%$ \\
\hline$>99$ & $27 / 38 ; 71.0 \%$ & $7 / 38 ; 18.4 \%$ & $18 / 38 ; 47.4 \%$ & $3 / 38 ; 7.9 \%$ & $19 / 32 ; 59.4 \%$ & $25 / 38 ; 65.8 \%$ \\
\hline
\end{tabular}


Figure 1. Major neuropathological comorbidities with ADD as compared to ADD as a sole diagnosis, by decade. The prevalences of all comorbidities shown increase with age, except for Lewy body disease.

\section{AD Comorbidity by Decade}

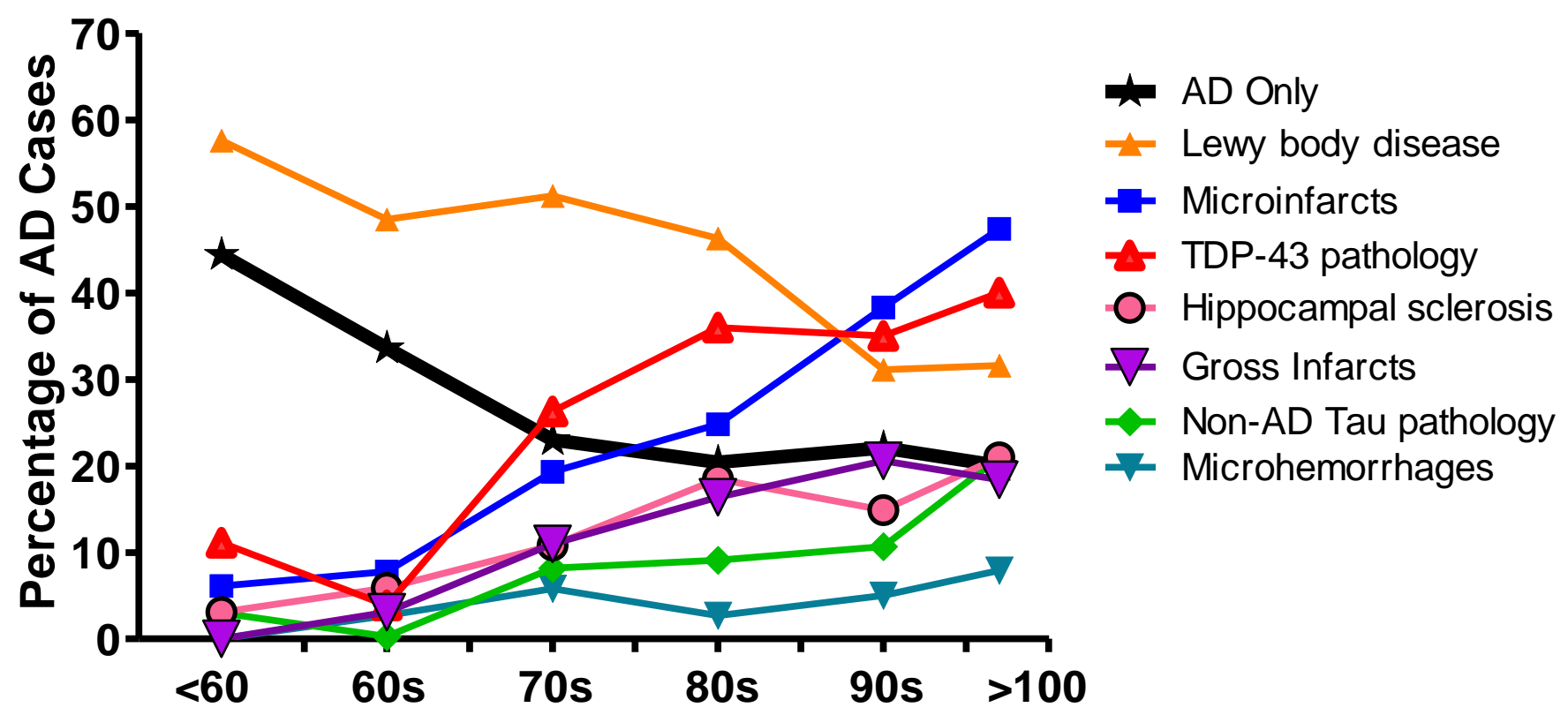




\section{Reference List}

[1] Zekry D, Duyckaerts C, Moulias R, Belmin J, Geoffre C, Herrmann F, Hauw JJ (2002) Degenerative and vascular lesions of the brain have synergistic effects in dementia of the elderly. Acta Neuropathol (Berl) 103, 481487.

[2] Jellinger KA , Attems J (2015) Challenges of multimorbidity of the aging brain: a critical update. J Neural Transm (Vienna) 122, 505-521.

[3] Beach TG, Adler CH, Sue LI, Serrano G, Shill HA, Walker DG, Lue L, Roher AE, Dugger BN, Maarouf C, Birdsill AC, Intorcia A, Saxon-Labelle M, Pullen J, Scroggins A, Filon J, Scott S, Hoffman B, Garcia A, Caviness JN, Hentz JG, Driver-Dunckley E, Jacobson SA, Davis KJ, Belden CM, Long KE, Malek-Ahmadi M, Powell JJ, Gale LD, Nicholson LR, Caselli RJ, Woodruff BK, Rapscak SZ, Ahern GL, Shi J, Burke AD, Reiman EM, Sabbagh MN (2015) Arizona Study of Aging and Neurodegenerative Disorders and Brain and Body Donation Program. Neuropathology 35, 354-389.

[4] Power MC, Mormino E, Soldan A, James BD, Yu L, Armstrong NM, Bangen KJ, Delano-Wood L, Lamar M, Lim YY, Nudelman K, Zahodne L, Gross AL, Mungas D, Widaman KF, Schneider J (2018) Combined neuropathological pathways account for age-related risk of dementia. Ann Neurol 84, 10-22.

[5] Neltner JH, Abner EL, Jicha GA, Schmitt FA, Patel E, Poon LW, Marla G, Green RC, Davey A, Johnson MA, Jazwinski SM, Kim S, Davis D, Woodard JL, Kryscio RJ, Van Eldik LJ, Nelson PT (2016) Brain pathologies in extreme old age. Neurobiol Aging 37, 1-11.

[6] Kovacs GG, Alafuzoff I, Al-Sarraj S, Arzberger T, Bogdanovic N, Capellari S, Ferrer I, Gelpi E, Kovari V, Kretzschmar H, Nagy Z, Parchi P, Seilhean D, Soininen H, Troakes C, Budka H (2008) Mixed brain pathologies in dementia: the BrainNet Europe consortium experience. Dement Geriatr Cogn Disord 26, 343-350.

[7] Rahimi J , Kovacs GG (2014) Prevalence of mixed pathologies in the aging brain. Alzheimers Res Ther 6, 82-

[8] Attems J , Jellinger K (2013) Neuropathological correlates of cerebral multimorbidity. Curr Alzheimer Res 10, 569577.

[9] Jellinger KA , Attems J (2007) Neuropathological evaluation of mixed dementia. J Neurol Sci 257, 80-87.

[10] Corrada MM, Sonnen JA, Kim RC, Kawas CH (2016) Microinfarcts are common and strongly related to dementia in the oldest-old: The 90+ study. Alzheimers Dement 12, 900-908.

[11] Kovari E, Gold G, Herrmann FR, Canuto A, Hof PR, Michel JP, Bouras C, Giannakopoulos P (2004) Cortical microinfarcts and demyelination significantly affect cognition in brain aging. Stroke 35, 410-414.

[12] Josephs KA, Whitwell JL, Weigand SD, Murray ME, Tosakulwong N, Liesinger AM, Petrucelli L, Senjem ML, Knopman DS, Boeve BF, Ivnik RJ, Smith GE, Jack CR, Jr., Parisi JE, Petersen RC, Dickson DW (2014) TDP-43 is a key player in the clinical features associated with Alzheimer's disease. Acta Neuropathol 127, 811824.

[13] James BD, Wilson RS, Boyle PA, Trojanowski JQ, Bennett DA, Schneider JA (2016) TDP-43 stage, mixed pathologies, and clinical Alzheimer's-type dementia. Brain 139, 2983-2993. 
[14] James BD, Bennett DA, Boyle PA, Leurgans S, Schneider JA (2012) Dementia from Alzheimer disease and mixed pathologies in the oldest old. JAMA 307, 1798-1800.

[15] Boyle PA, Yu L, Leurgans SE, Wilson RS, Brookmeyer R, Schneider JA, Bennett DA (2019) Attributable risk of Alzheimer's dementia attributed to age-related neuropathologies. Ann Neurol 85, 114-124.

[16] Boyle PA, Yang J, Yu L, Leurgans SE, Capuano AW, Schneider JA, Wilson RS, Bennett DA (2017) Varied effects of age-related neuropathologies on the trajectory of late life cognitive decline. Brain 140, 804-812.

[17] Schneider JA, Arvanitakis Z, Bang W, Bennett DA (2007) Mixed brain pathologies account for most dementia cases in community-dwelling older persons. Neurology 69, 2197-2204.

[18] Boyle PA, Wilson RS, Yu L, Barr AM, Honer WG, Schneider JA, Bennett DA (2013) Much of late life cognitive decline is not due to common neurodegenerative pathologies. Ann Neurol 74, 478-489.

[19] Farfel JM, Yu L, Boyle PA, Leurgans S, Shah RC, Schneider JA, Bennett DA (2019) Alzheimer's disease frequency peaks in the tenth decade and is lower afterwards. Acta Neuropathol Commun 7, 104-

[20] Kapasi A, DeCarli C, Schneider JA (2017) Impact of multiple pathologies on the threshold for clinically overt dementia. Acta Neuropathol 134, 171-186.

[21] Jansen WJ, Wilson RS, Visser PJ, Nag S, Schneider JA, James BD, Leurgans SE, Capuano AW, Bennett DA, Boyle PA (2018) Age and the association of dementia-related pathology with trajectories of cognitive decline. Neurobiol Aging 61, 138-145.

[22] Robinson JL, Corrada MM, Kovacs GG, Dominique M, Caswell C, Xie SX, Lee VM, Kawas CH, Trojanowski JQ (2018) Non-Alzheimer's contributions to dementia and cognitive resilience in The 90+ Study. Acta Neuropathol 136, 377-388.

[23] Malek-Ahmadi M, Beach TG, Zamrini E, Adler CH, Sabbagh MN, Shill HA, Jacobson SA, Belden CM, Caselli RJ, Woodruff BK, Rapscak SZ, Ahern GL, Shi J, Caviness JN, Driver-Dunckley E, Mehta SH, Shprecher DR, Spann BM, Tariot P, Davis KJ, Long KE, Nicholson LR, Intorcia A, Glass MJ, Walker JE, Callan M, Curry J, Cutler B, Oliver J, Arce R, Walker DG, Lue LF, Serrano GE, Sue LI, Chen K, Reiman EM (2019) Faster cognitive decline in dementia due to Alzheimer disease with clinically undiagnosed Lewy body disease. PLoS One 14, e0217566-

[24] Sonnen JA, Santa CK, Hemmy LS, Woltjer R, Leverenz JB, Montine KS, Jack CR, Kaye J, Lim K, Larson EB, White L, Montine TJ (2011) Ecology of the aging human brain. Arch Neurol 68, 1049-1056.

[25] Brenowitz WD, Keene CD, Hawes SE, Hubbard RA, Longstreth WT, Jr., Woltjer RL, Crane PK, Larson EB, Kukull WA (2017) Alzheimer's disease neuropathologic change, Lewy body disease, and vascular brain injury in clinic- and community-based samples. Neurobiol Aging 53, 83-92.

[26] Wilson RS, Capuano AW, Bennett DA, Schneider JA, Boyle PA (2016) Temporal course of neurodegenerative effects on cognition in old age. Neuropsychology 30, 591-599.

[27] Caselli RJ, Beach TG, Knopman DS, Graff-Radford NR (2017) Alzheimer Disease: Scientific Breakthroughs and Translational Challenges. Mayo Clin Proc 92, 978-994. 
[28] White LR, Edland SD, Hemmy LS, Montine KS, Zarow C, Sonnen JA, Uyehara-Lock JH, Gelber RP, Ross GW, Petrovitch H, Masaki KH, Lim KO, Launer LJ, Montine TJ (2016) Neuropathologic comorbidity and cognitive impairment in the Nun and Honolulu-Asia Aging Studies. Neurology 86, 1000-1008.

[29] Kawas CH, Kim RC, Sonnen JA, Bullain SS, Trieu T, Corrada MM (2015) Multiple pathologies are common and related to dementia in the oldest-old: The 90+ Study. Neurology 85, 535-542.

[30] Sonnen JA, Crane PK, Haneuse S, Li G, Schellenberg GD, Craft S, Montine TJ (2007) Pathological correlates of dementia in a longitudinal, population-based sample of aging. Ann Neurol 62, 406-413.

[31] Bennett DA, Schneider JA, Arvanitakis Z, Kelly JF, Aggarwal NT, Shah RC, Wilson RS (2006) Neuropathology of older persons without cognitive impairment from two community-based studies. Neurology 66, 18371844.

[32] Negash S, Bennett DA, Wilson RS, Schneider JA, Arnold SE (2011) Cognition and neuropathology in aging: multidimensional perspectives from the Rush Religious Orders Study and Rush Memory And Aging Project. Curr Alzheimer Res 8, 336-340.

[33] Dugger BN, Clark CM, Serrano G, Mariner M, Bedell BJ, Coleman RE, Doraiswamy PM, Lu M, Fleisher AS, Reiman EM, Sabbagh MN, Sadowsky CH, Schneider JA, Zehntner SP, Carpenter AP, Joshi AD, Mintun MA, Pontecorvo MJ, Skovronsky DM, Sue LI, Beach TG (2014) Neuropathologic heterogeneity does not impair florbetapir-positron emission tomography postmortem correlates. J Neuropathol Exp Neurol 73, 72-80.

[34] Serrano GE, Sabbagh MN, Sue LI, Hidalgo JA, Schneider JA, Bedell BJ, Van Deerlin VM, Suh E, Akiyama H, Joshi $A D$, Pontecorvo MJ, Mintun MA, Beach TG (2014) Positive florbetapir PET amyloid imaging in a subject with frequent cortical neuritic plaques and frontotemporal lobar degeneration with TDP43-positive inclusions. J Alzheimers Dis 42, 813-821.

[35] Arvanitakis Z, Leurgans SE, Barnes LL, Bennett DA, Schneider JA (2011) Microinfarct pathology, dementia, and cognitive systems. Stroke 42, 722-727.

[36] Nag S, Yu L, Boyle PA, Leurgans SE, Bennett DA, Schneider JA (2018) TDP-43 pathology in anterior temporal pole cortex in aging and Alzheimer's disease. Acta Neuropathol Commun 6, 33-

[37] Nelson PT, Abner EL, Schmitt FA, Kryscio RJ, Jicha GA, Smith CD, Davis DG, Poduska JW, Patel E, Mendiondo MS, Markesbery WR (2010) Modeling the association between 43 different clinical and pathological variables and the severity of cognitive impairment in a large autopsy cohort of elderly persons. Brain Pathol 20, 66-79.

[38] Smith VD, Bachstetter AD, Ighodaro E, Roberts K, Abner EL, Fardo DW, Nelson PT (2018) Overlapping but distinct TDP-43 and tau pathologic patterns in aged hippocampi. Brain Pathol 28, 264-273.

[39] Josephs KA, Dickson DW, Tosakulwong N, Weigand SD, Murray ME, Petrucelli L, Liesinger AM, Senjem ML, Spychalla AJ, Knopman DS, Parisi JE, Petersen RC, Jack CR, Jr., Whitwell JL (2017) Rates of hippocampal atrophy and presence of post-mortem TDP-43 in patients with Alzheimer's disease: a longitudinal retrospective study. Lancet Neurol 16, 917-924.

[40] Josephs KA, Whitwell JL, Weigand SD, Murray ME, Tosakulwong N, Liesinger AM, Petrucelli L, Senjem ML, Knopman DS, Boeve BF, Ivnik RJ, Smith GE, Jack CR, Jr., Parisi JE, Petersen RC, Dickson DW (2014) TDP-43 
is a key player in the clinical features associated with Alzheimer's disease. Acta Neuropathol 127, 811824.

[41] Malek-Ahmadi M (2019) Faster cognitive decline in dementia due to Alzheimer's disease with clinically undiagnosed Lewy body disease. PLoS One in press

[42] Olichney JM, Galasko D, Salmon DP, Hofstetter CR, Hansen LA, Katzman R, Thal LJ (1998) Cognitive decline is faster in Lewy body variant than in Alzheimer's disease. Neurology 51, 351-357.

[43] Kramberger MG, Auestad B, Garcia-Ptacek S, Abdelnour C, Olmo JG, Walker Z, Lemstra AW, Londos E, Blanc F, Bonanni L, McKeith I, Winblad B, de Jong FJ, Nobili F, Stefanova E, Petrova M, Falup-Pecurariu C, Rektorova I, Bostantjopoulou S, Biundo R, Weintraub D, Aarsland D (2017) Long-Term Cognitive Decline in Dementia with Lewy Bodies in a Large Multicenter, International Cohort. J Alzheimers Dis 57, 787-795.

[44] Brenowitz WD, Hubbard RA, Keene CD, Hawes SE, Longstreth WT, Jr., Woltjer RL, Kukull WA (2017) Mixed neuropathologies and estimated rates of clinical progression in a large autopsy sample. Alzheimers Dement 13, 654-662.

[45] Kraybill ML, Larson EB, Tsuang DW, Teri L, McCormick WC, Bowen JD, Kukull WA, Leverenz JB, Cherrier MM (2005) Cognitive differences in dementia patients with autopsy-verified AD, Lewy body pathology, or both. Neurology 64, 2069-2073.

[46] Haneuse S, Larson E, Walker R, Montine T, Sonnen J (2009) Neuropathology-based risk scoring for dementia diagnosis in the elderly. J Alzheimers Dis 17, 875-885.

[47] Boyle PA, Yu L, Wilson RS, Leurgans SE, Schneider JA, Bennett DA (2018) Person-specific contribution of neuropathologies to cognitive loss in old age. Ann Neurol 83, 74-83.

[48] Lippa CF , Dickson DW (2004) Hippocampal sclerosis dementia: expanding the phenotypes of frontotemporal dementias? Neurology 63, 414-415.

[49] Dutra JR, Cortes EP, Vonsattel JP (2015) Update on Hippocampal Sclerosis. Curr Neurol Neurosci Rep 15, 67-

[50] Nelson PT, Trojanowski JQ, Abner EL, Al-Janabi OM, Jicha GA, Schmitt FA, Smith CD, Fardo DW, Wang WX, Kryscio RJ, Neltner JH, Kukull WA, Cykowski MD, Van Eldik LJ, Ighodaro ET (2016) "New Old Pathologies": AD, PART, and Cerebral Age-Related TDP-43 With Sclerosis (CARTS). J Neuropathol Exp Neurol 75, 482498.

[51] Nelson PT, Smith CD, Abner EL, Wilfred BJ, Wang WX, Neltner JH, Baker M, Fardo DW, Kryscio RJ, Scheff SW, Jicha GA, Jellinger KA, Van Eldik L, Schmitt FA (2013) Hippocampal sclerosis of aging, a prevalent and high-morbidity brain disease. Acta Neuropathol 126, 161-177.

[52] Nelson PT, Head E, Schmitt FA, Davis PR, Neltner JH, Jicha GA, Abner EL, Smith CD, Van Eldik LJ, Kryscio RJ, Scheff SW (2011) Alzheimer's disease is not "brain aging": neuropathological, genetic, and epidemiological human studies. Acta Neuropathol 121, 571-587.

[53] Sahoo A, Bejanin A, Murray ME, Tosakulwong N, Weigand SD, Serie AM, Senjem ML, Machulda MM, Parisi JE, Boeve BF, Knopman DS, Petersen RC, Dickson DW, Whitwell JL, Josephs KA (2018) TDP-43 and Alzheimer's Disease Pathologic Subtype in Non-Amnestic Alzheimer's Disease Dementia. J Alzheimers Dis 64, 1227-1233. 
[54] Keage HA, Hunter S, Matthews FE, Ince PG, Hodges J, Hokkanen SR, Highley JR, Dening T, Brayne C (2014) TDP43 pathology in the population: prevalence and associations with dementia and age. J Alzheimers Dis $\mathbf{4 2 ,}$ 641-650.

[55] Josephs KA, Whitwell JL, Tosakulwong N, Weigand SD, Murray ME, Liesinger AM, Petrucelli L, Senjem ML, Ivnik RJ, Parisi JE, Petersen RC, Dickson DW (2015) TAR DNA-binding protein 43 and pathological subtype of Alzheimer's disease impact clinical features. Ann Neurol 78, 697-709.

[56] Nag S, Yu L, Wilson RS, Chen EY, Bennett DA, Schneider JA (2017) TDP-43 pathology and memory impairment in elders without pathologic diagnoses of AD or FTLD. Neurology 88, 653-660.

[57] Kapasi A, Yu L, Stewart CC, Schneider JA, Bennett DA, Boyle PA (2019) Association of TDP-43 Pathology With Domain-specific Literacy in Older Persons. Alzheimer Dis Assoc Disord

[58] Gold G, Giannakopoulos P, Herrmann FR, Bouras C, Kovari E (2007) Identification of Alzheimer and vascular lesion thresholds for mixed dementia. Brain 130, 2830-2836.

[59] Hall A, Pekkala T, Polvikoski T, van GM, Kivipelto M, Lotjonen J, Mattila J, Kero M, Myllykangas L, Makela M, Oinas M, Paetau A, Soininen H, Tanskanen M, Solomon A (2019) Prediction models for dementia and neuropathology in the oldest old: the Vantaa 85+ cohort study. Alzheimers Res Ther 11, 11-

[60] Tanskanen M, Makela M, Notkola IL, Myllykangas L, Rastas S, Oinas M, Lindsberg PJ, Polvikoski T, Tienari PJ, Paetau A (2017) Population-based analysis of pathological correlates of dementia in the oldest old. Ann Clin Transl Neurol 4, 154-165.

[61] Kapasi A, Leurgans SE, James BD, Boyle PA, Arvanitakis Z, Nag S, Bennett DA, Buchman AS, Schneider JA (2018) Watershed microinfarct pathology and cognition in older persons. Neurobiol Aging 70, 10-17.

[62] Kryscio RJ, Abner EL, Nelson PT, Bennett D, Schneider J, Yu L, Hemmy LS, Lim KO, Masaki K, Cairns N, Xiong C, Woltjer R, Dodge HH, Tyas S, Fardo DW, Lou W, Wan L, Schmitt FA (2016) The Effect of Vascular Neuropathology on Late-life Cognition: Results from the SMART Project. J Prev Alzheimers Dis 3, 85-91.

[63] Erkinjuntti T, Haltia M, Palo J, Sulkava R, Paetau A (1988) Accuracy of the clinical diagnosis of vascular dementia: a prospective clinical and post-mortem neuropathological study. J Neurol Neurosurg Psychiatry 51, 1037-1044.

[64] del Ser T, Bermejo F, Portera A, Arredondo JM, Bouras C, Constantinidis J (1990) Vascular dementia. A clinicopathological study. J Neurol Sci 96, 1-17.

[65] Schneider JA, Wilson RS, Cochran EJ, Bienias JL, Arnold SE, Evans DA, Bennett DA (2003) Relation of cerebral infarctions to dementia and cognitive function in older persons. Neurology 60, 1082-1088.

[66] Schneider JA, Wilson RS, Bienias JL, Evans DA, Bennett DA (2004) Cerebral infarctions and the likelihood of dementia from Alzheimer disease pathology. Neurology 62, 1148-1155.

[67] Jellinger KA (2002) The pathology of ischemic-vascular dementia: an update. J Neurol Sci 203-204, 153-157.

[68] Esiri MM (2000) Which vascular lesions are of importance in vascular dementia? Ann N Y Acad Sci 903, 239-243. 
[69] van Veluw SJ, Shih AY, Smith EE, Chen C, Schneider JA, Wardlaw JM, Greenberg SM, Biessels GJ (2017) Detection, risk factors, and functional consequences of cerebral microinfarcts. Lancet Neurol 16, 730-740.

[70] Kalback W, Esh C, Castano EM, Rahman A, Kokjohn T, Luehrs DC, Sue L, Cisneros R, Gerber F, Richardson C, Bohrmann B, Walker DG, Beach TG, Roher AE (2004) Atherosclerosis, vascular amyloidosis and brain hypoperfusion in the pathogenesis of sporadic Alzheimer's disease. Neurol Res 26, 525-539.

[71] Beach TG, Adler CH, Sue LI, Serrano G, Shill HA, Walker DG, Lue L, Roher AE, Dugger BN, Maarouf C, Birdsill AC, Intorcia A, Saxon-Labelle M, Pullen J, Scroggins A, Filon J, Scott S, Hoffman B, Garcia A, Caviness JN, Hentz JG, Driver-Dunckley E, Jacobson SA, Davis KJ, Belden CM, Long KE, Malek-Ahmadi M, Powell JJ, Gale LD, Nicholson LR, Caselli RJ, Woodruff BK, Rapscak SZ, Ahern GL, Shi J, Burke AD, Reiman EM, Sabbagh MN (2015) Arizona Study of Aging and Neurodegenerative Disorders and Brain and Body Donation Program. Neuropathology

[72] Besser L, Kukull W, Knopman DS, Chui H, Galasko D, Weintraub S, Jicha G, Carlsson C, Burns J, Quinn J, Sweet RA, Rascovsky K, Teylan M, Beekly D, Thomas G, Bollenbeck M, Monsell S, Mock C, Zhou XH, Thomas N, Robichaud E, Dean M, Hubbard J, Jacka M, Schwabe-Fry K, Wu J, Phelps C, Morris JC (2018) Version 3 of the National Alzheimer's Coordinating Center's Uniform Data Set. Alzheimer Dis Assoc Disord 32, 351358.

[73] Besser LM, Kukull WA, Teylan MA, Bigio EH, Cairns NJ, Kofler JK, Montine TJ, Schneider JA, Nelson PT (2018) The Revised National Alzheimer's Coordinating Center's Neuropathology Form-Available Data and New Analyses. J Neuropathol Exp Neurol 77, 717-726.

[74] Hyman BT, Phelps CH, Beach TG, Bigio EH, !Lost Data, Carrillo MC, Dickson DW, Duyckaerts C, Frosch MP, Masliah E, Mirra SS, Nelson PT, Schneider JA, Thal DR, Thies B, Trojanowski JQ, Vinters HV, Montine TJ (2012) National Institute on Aging-Alzheimer's Association guidelines for the neuropathologic assessment of Alzheimer's disease. Alzheimers Dement 8, 1-13.

[75] Montine TJ, Phelps CH, Beach TG, Bigio EH, Cairns NJ, Dickson DW, Duyckaerts C, Frosch MP, Masliah E, Mirra SS, Nelson PT, Schneider JA, Thal DR, Trojanowski JQ, Vinters HV, Hyman BT (2012) National Institute on Aging-Alzheimer's Association guidelines for the neuropathologic assessment of Alzheimer's disease: a practical approach. Acta Neuropathol 123, 1-11.

[76] Thal DR, Rub U, Orantes M, Braak H (2002) Phases of A beta-deposition in the human brain and its relevance for the development of AD. Neurology 58, 1791-1800.

[77] Alafuzoff I, Arzberger T, Al-Sarraj S, Bodi I, Bogdanovic N, Braak H, Bugiani O, Del-Tredici K, Ferrer I, Gelpi E, Giaccone G, Graeber MB, Ince P, Kamphorst W, King A, Korkolopoulou P, Kovacs GG, Larionov S, Meyronet D, Monoranu C, Parchi P, Patsouris E, Roggendorf W, Seilhean D, Tagliavini F, Stadelmann C, Streichenberger N, Thal DR, Wharton SB, Kretzschmar H (2008) Staging of neurofibrillary pathology in Alzheimer's disease: a study of the BrainNet Europe Consortium. Brain Pathol 18, 484-496.

[78] Braak H, Alafuzoff I, Arzberger T, Kretzschmar H, Del TK (2006) Staging of Alzheimer disease-associated neurofibrillary pathology using paraffin sections and immunocytochemistry. Acta Neuropathol 112, 389404.

[79] Mirra SS, Heyman A, McKeel D, Sumi SM, Crain BJ, Brownlee LM, Vogel FS, Hughes JP, van Belle G, Berg L (1991) The Consortium to Establish a Registry for Alzheimer's Disease (CERAD). Part II. Standardization of the neuropathologic assessment of Alzheimer's disease. Neurology 41, 479-486. 
[80] Nelson PT, Dickson DW, Trojanowski JQ, Jack CR, Boyle PA, Arfanakis K, Rademakers R, Alafuzoff I, Attems J, Brayne C, Coyle-Gilchrist ITS, Chui HC, Fardo DW, Flanagan ME, Halliday G, Hokkanen SRK, Hunter S, Jicha GA, Katsumata Y, Kawas CH, Keene CD, Kovacs GG, Kukull WA, Levey Al, Makkinejad N, Montine TJ, Murayama S, Murray ME, Nag S, Rissman RA, Seeley WW, Sperling RA, White lii CL, Yu L, Schneider JA (2019) Limbic-predominant age-related TDP-43 encephalopathy (LATE): consensus working group report. Brain 142, 1503-1527.

[81] Yang Z, Slavin MJ, Sachdev PS (2013) Dementia in the oldest old. Nat Rev Neurol 9, 382-393.

[82] Halliday G, Brooks W, Arthur H, Creasey H, Broe GA (1997) Further evidence for an association between a mutation in the APP gene and Lewy body formation. Neurosci Lett 227, 49-52.

[83] Ringman JM, Monsell S, Ng DW, Zhou Y, Nguyen A, Coppola G, Van B, V, Mendez MF, Tung S, Weintraub S, Mesulam MM, Bigio EH, Gitelman DR, Fisher-Hubbard AO, Albin RL, Vinters HV (2016) Neuropathology of Autosomal Dominant Alzheimer Disease in the National Alzheimer Coordinating Center Database. $J$ Neuropathol Exp Neurol 75, 284-290.

[84] Ishikawa A, Piao YS, Miyashita A, Kuwano R, Onodera O, Ohtake H, Suzuki M, Nishizawa M, Takahashi H (2005) A mutant PSEN1 causes dementia with Lewy bodies and variant Alzheimer's disease. Ann Neurol 57, 429434.

[85] Rosenberg CK, Pericak-Vance MA, Saunders AM, Gilbert JR, Gaskell PC, Hulette CM (2000) Lewy body and Alzheimer pathology in a family with the amyloid-beta precursor protein APP717 gene mutation. Acta Neuropathol 100, 145-152.

[86] Lantos PL, Ovenstone IM, Johnson J, Clelland CA, Roques P, Rossor MN (1994) Lewy bodies in the brain of two members of a family with the 717 (Val to lle) mutation of the amyloid precursor protein gene. Neurosci Lett 172, 77-79.

[87] Lippa CF, Fujiwara H, Mann DM, Giasson B, Baba M, Schmidt ML, Nee LE, O'Connell B, Pollen DA, St GeorgeHyslop P, Ghetti B, Nochlin D, Bird TD, Cairns NJ, Lee VM, Iwatsubo T, Trojanowski JQ (1998) Lewy bodies contain altered alpha-synuclein in brains of many familial Alzheimer's disease patients with mutations in presenilin and amyloid precursor protein genes. Am J Pathol 153, 1365-1370.

[88] Leverenz JB, Fishel MA, Peskind ER, Montine TJ, Nochlin D, Steinbart E, Raskind MA, Schellenberg GD, Bird TD, Tsuang D (2006) Lewy body pathology in familial Alzheimer disease: evidence for disease- and mutationspecific pathologic phenotype. Arch Neurol 63, 370-376.

[89] Kaneko H, Kakita A, Kasuga K, Nozaki H, Ishikawa A, Miyashita A, Kuwano R, Ito G, Iwatsubo T, Takahashi H, Nishizawa M, Onodera O, Sisodia SS, Ikeuchi T (2007) Enhanced accumulation of phosphorylated alphasynuclein and elevated beta-amyloid 42/40 ratio caused by expression of the presenilin-1 deltaT440 mutant associated with familial Lewy body disease and variant Alzheimer's disease. J Neurosci 27, 13092-13097.

[90] Haltia M, Ghiso J, Wisniewski T, Kiuru S, Miller D, Frangione B (1991) Gelsolin variant and beta-amyloid co-occur in a case of Alzheimer's with Lewy bodies. Neurobiol Aging 12, 313-316.

[91] Bugiani O, Giaccone G, Piccardo P, Morbin M, Tagliavini F, Ghetti B (2000) Neuropathology of GerstmannStraussler-Scheinker disease. Microsc Res Tech 50, 10-15. 
[92] Tsuang DW, Riekse RG, Purganan KM, David AC, Montine TJ, Schellenberg GD, Steinbart EJ, Petrie EC, Bird TD (2006) Lewy body pathology in late-onset familial Alzheimer's disease: a clinicopathological case series. J Alzheimers Dis 9, 235-242.

[93] Uchikado H, !Lost Data, DeLucia MW, Dickson DW (2006) Alzheimer disease with amygdala Lewy bodies: a distinct form of alpha-synucleinopathy. J Neuropathol Exp Neurol 65, 685-697.

[94] Hamilton RL (2000) Lewy bodies in Alzheimer's disease: a neuropathological review of 145 cases using alphasynuclein immunohistochemistry. Brain Pathol 10, 378-384.

[95] Bertrand E, Lechowicz W, Szpak GM, Lewandowska E, Dymecki J, Wierzba-Bobrowicz T (2004) Limbic neuropathology in idiopathic Parkinson's disease with concomitant dementia. Folia Neuropathol 42, 141-150.

[96] Kovari E, Gold G, Herrmann FR, Canuto A, Hof PR, Bouras C, Giannakopoulos P (2003) Lewy body densities in the entorhinal and anterior cingulate cortex predict cognitive deficits in Parkinson's disease. Acta Neuropathol (Berl) 106, 83-88.

[97] Mattila PM, Rinne JO, Helenius H, Dickson DW, Roytta M (2000) Alpha-synuclein-immunoreactive cortical Lewy bodies are associated with cognitive impairment in Parkinson's disease. Acta Neuropathol (Berl) 100, 285-290.

[98] Hurtig HI, Trojanowski JQ, Galvin J, Ewbank D, Schmidt ML, Lee VM, Clark CM, Glosser G, Stern MB, Gollomp SM, Arnold SE (2000) Alpha-synuclein cortical Lewy bodies correlate with dementia in Parkinson's disease. Neurology 54, 1916-1921.

[99] Mattila PM, Rinne JO, Helenius H, Dickson DW, Roytta M (2000) Alpha-synuclein-immunoreactive cortical Lewy bodies are associated with cognitive impairment in Parkinson's disease. Acta Neuropathol (Berl) 100, 285-290.

[100] Braak H, Rub U, Jansen Steur EN, Del Tredici K, de Vos RA (2005) Cognitive status correlates with neuropathologic stage in Parkinson disease. Neurology 64, 1404-1410.

[101] Jellinger KA (2009) Significance of brain lesions in Parkinson disease dementia and Lewy body dementia. Front Neurol Neurosci 24, 114-125.

[102] Burack MA, Hartlein J, Flores HP, Taylor-Reinwald L, Perlmutter JS, Cairns NJ (2010) In vivo amyloid imaging in autopsy-confirmed Parkinson disease with dementia. Neurology 74, 77-84.

[103] Irwin DJ, White MT, Toledo JB, Xie SX, Robinson JL, Van D, V, Lee VM, Leverenz JB, Montine TJ, Duda JE, Hurtig HI, Trojanowski JQ (2012) Neuropathologic substrates of Parkinson disease dementia. Ann Neurol 72, 587-598.

[104] Sabbagh MN, Adler CH, Lahti TJ, Connor DJ, Vedders L, Peterson LK, Caviness JN, Shill HA, Sue LI, Ziabreva I, Perry E, Ballard CG, Aarsland D, Walker DG, Beach TG (2009) Parkinson disease with dementia: comparing patients with and without Alzheimer pathology. Alzheimer Dis Assoc Disord 23, 295-297.

[105] Compta Y, Parkkinen L, O'Sullivan SS, Vandrovcova J, Holton JL, Collins C, Lashley T, Kallis C, Williams DR, de SR, Lees AJ, Revesz T (2011) Lewy- and Alzheimer-type pathologies in Parkinson's disease dementia: which is more important? Brain 134, 1493-1505. 
[106] Hely MA, Reid WG, Adena MA, Halliday GM, Morris JG (2008) The Sydney multicenter study of Parkinson's disease: the inevitability of dementia at 20 years. Mov Disord 23, 837-844.

[107] Akhtar RS, Xie SX, Brennan L, Pontecorvo MJ, Hurtig HI, Trojanowski JQ, Weintraub D, Siderowf AD (2016) Amyloid-Beta Positron Emission Tomography Imaging of Alzheimer's Pathology in Parkinson's Disease Dementia. Mov Disord Clin Pract 3, 367-375.

[108] Deramecourt V, Bombois S, Maurage CA, Ghestem A, Drobecq H, Vanmechelen E, Lebert F, Pasquier F, Delacourte $A$ (2006) Biochemical staging of synucleinopathy and amyloid deposition in dementia with Lewy bodies. J Neuropathol Exp Neurol 65, 278-288.

[109] Graff-Radford J, Aakre J, Savica R, Boeve B, Kremers WK, Ferman TJ, Jones DT, Kantarci K, Knopman DS, Dickson DW, Kukull WA, Petersen RC (2017) Duration and Pathologic Correlates of Lewy Body Disease. JAMA Neurol 74, 310-315.

[110] Barker WW, Luis CA, Kashuba A, Luis M, Harwood DG, Loewenstein D, Waters C, Jimison P, Shepherd E, Sevush S, Graff-Radford N, Newland D, Todd M, Miller B, Gold M, Heilman K, Doty L, Goodman I, Robinson B, Pearl G, Dickson D, Duara R (2002) Relative frequencies of Alzheimer disease, Lewy body, vascular and frontotemporal dementia, and hippocampal sclerosis in the State of Florida Brain Bank. Alzheimer Dis Assoc Disord 16, 203-212.

[111] Beach TG, Adler CH, Sue LI, Serrano G, Shill HA, Walker DG, Lue L, Roher AE, Dugger BN, Maarouf C, Birdsill AC, Intorcia A, Saxon-Labelle M, Pullen J, Scroggins A, Filon J, Scott S, Hoffman B, Garcia A, Caviness JN, Hentz JG, Driver-Dunckley E, Jacobson SA, Davis KJ, Belden CM, Long KE, Malek-Ahmadi M, Powell JJ, Gale LD, Nicholson LR, Caselli RJ, Woodruff BK, Rapscak SZ, Ahern GL, Shi J, Burke AD, Reiman EM, Sabbagh MN (2015) Arizona Study of Aging and Neurodegenerative Disorders and Brain and Body Donation Program. Neuropathology 35, 354-389.

[112] Beach TG, Adler CH, Zhang N, Serrano GE, Sue LI, Driver-Dunckley E, Metha SH, Zamrini E, Sabbagh NMN, Shill HA, Belden CM, Shprecher DR, aselli RJ, Reiman EM, Davis KJ, Long KE, Nicholson LR, Intorcia AJ, Glass MJ, Walker JE, Callan M, Oliver JC, Arce R, Gerkin RC (2019) Severe hyposmia distinguishes neuropathologically-confirmed dementia with Lewy bodies from Alzheimer's disease dementia. BIORXIV 743856; doi: https://doi.org/10.1101/743856

[113] McKeith I, Taylor JP, Thomas A, Donaghy P, Kane J (2016) Revisiting DLB Diagnosis: A Consideration of Prodromal DLB and of the Diagnostic Overlap With Alzheimer Disease. J Geriatr Psychiatry Neurol 29, 249-253.

[114] Lebouvier T, Delrieu J, Evain S, Pallardy A, Sauvaget A, Letournel F, Chevrier R, Lepetit M, Vercelletto M, Boutoleau-Bretonniere C, Derkinderen P (2013) [Dementia: Where are the Lewy bodies?]. Rev Neurol (Paris) 169, 844-857.

[115] Nelson PT, Jicha GA, Kryscio RJ, Abner EL, Schmitt FA, Cooper G, Xu LO, Smith CD, Markesbery WR (2010) Low sensitivity in clinical diagnoses of dementia with Lewy bodies. J Neurol 257, 359-366.

[116] Ferman TJ, Aoki N, Crook JE, Murray ME, Graff-Radford NR, van Gerpen JA, Uitti RJ, Wszolek ZK, Graff-Radford J, Pedraza O, Kantarci K, Boeve BF, Dickson DW (2018) The limbic and neocortical contribution of alphasynuclein, tau, and amyloid beta to disease duration in dementia with Lewy bodies. Alzheimers Dement 14, 330-339. 
[117] Beach TG, Adler CH, Lue L, Sue LI, Bachalakuri J, Henry-Watson J, Sasse J, Boyer S, Shirohi S, Brooks R, Eschbacher J, !Lost Data, Akiyama H, Caviness J, Shill HA, Connor DJ, Sabbagh MN, Walker DG (2009) Unified staging system for Lewy body disorders: correlation with nigrostriatal degeneration, cognitive impairment and motor dysfunction. Acta Neuropathol 117, 613-634.

[118] McKeith IG, Dickson DW, Lowe J, Emre M, O'Brien JT, Feldman H, Cummings J, Duda JE, Lippa C, Perry EK, Aarsland D, Arai H, Ballard CG, Boeve B, Burn DJ, Costa D, Del ST, Dubois B, Galasko D, Gauthier S, Goetz CG, Gomez-Tortosa E, Halliday G, Hansen LA, Hardy J, Iwatsubo T, Kalaria RN, Kaufer D, Kenny RA, Korczyn A, Kosaka K, Lee VM, Lees A, Litvan I, Londos E, Lopez OL, Minoshima S, Mizuno Y, Molina JA, Mukaetova-Ladinska EB, Pasquier F, Perry RH, Schulz JB, Trojanowski JQ, Yamada M (2005) Diagnosis and management of dementia with Lewy bodies: third report of the DLB Consortium. Neurology 65, 1863-1872.

[119] McKeith IG, Boeve BF, Dickson DW, Halliday G, Taylor JP, Weintraub D, Aarsland D, Galvin J, Attems J, Ballard CG, Bayston A, Beach TG, Blanc F, Bohnen N, Bonanni L, Bras J, Brundin P, Burn D, Chen-Plotkin A, Duda JE, El-Agnaf O, Feldman H, Ferman TJ, Ffytche D, Fujishiro H, Galasko D, Goldman JG, Gomperts SN, GraffRadford NR, Honig LS, Iranzo A, Kantarci K, Kaufer D, Kukull W, Lee VMY, Leverenz JB, Lewis S, Lippa C, Lunde A, Masellis M, Masliah E, McLean P, Mollenhauer B, Montine TJ, Moreno E, Mori E, Murray M, O'Brien JT, Orimo S, Postuma RB, Ramaswamy S, Ross OA, Salmon DP, Singleton A, Taylor A, Thomas A, Tiraboschi P, Toledo JB, Trojanowski JQ, Tsuang D, Walker Z, Yamada M, Kosaka K (2017) Diagnosis and management of dementia with Lewy bodies: Fourth consensus report of the DLB Consortium. Neurology 89, 88-100.

[120] Savica R, Beach TG, Hentz JG, Sabbagh MN, Serrano GE, Sue LI, Dugger BN, Shill HA, Driver-Dunckley E, Caviness JN, Mehta SH, Jacobson SA, Belden CM, Davis KJ, Zamrini E, Shprecher DR, Adler CH (2018) Lewy Body P:athology in Alzheimer's Disease: A Clinicopathological Prospective Study. Acta Neurol Scand

[121] Brun A , Englund E (1986) A white matter disorder in dementia of the Alzheimer type: a pathoanatomical study. Ann Neurol 19, 253-262.

[122] Englund E, Brun A, Alling C (1988) White matter changes in dementia of Alzheimer's type. Biochemical and neuropathological correlates. Brain 111, 1425-1439.

[123] Mirsen TR, Lee DH, Wong CJ, Diaz JF, Fox AJ, Hachinski VC, Merskey H (1991) Clinical correlates of white-matter changes on magnetic resonance imaging scans of the brain. Arch Neurol 48, 1015-1021.

[124] Prins ND , Scheltens P (2015) White matter hyperintensities, cognitive impairment and dementia: an update. Nat Rev Neurol 11, 157-165.

[125] Leys D, Pruvo JP, Parent M, Vermersch P, Soetaert G, Steinling M, Delacourte A, Defossez A, Rapoport A, Clarisse J, . (1991) Could Wallerian degeneration contribute to "leuko-araiosis" in subjects free of any vascular disorder? J Neurol Neurosurg Psychiatry 54, 46-50.

[126] Ihara M, Polvikoski TM, Hall R, Slade JY, Perry RH, Oakley AE, Englund E, O'Brien JT, Ince PG, Kalaria RN (2010) Quantification of myelin loss in frontal lobe white matter in vascular dementia, Alzheimer's disease, and dementia with Lewy bodies. Acta Neuropathol 119, 579-589.

[127] Polvikoski TM, van Straaten EC, Barkhof F, Sulkava R, Aronen HJ, Niinisto L, Oinas M, Scheltens P, Erkinjuntti T, Kalaria RN (2010) Frontal lobe white matter hyperintensities and neurofibrillary pathology in the oldest old. Neurology 75, 2071-2078. 
[128] Alhassoon OM, Sorg SF, Taylor MJ, Stephan RA, Schweinsburg BC, Stricker NH, Gongvatana A, Grant I (2012) Callosal white matter microstructural recovery in abstinent alcoholics: a longitudinal diffusion tensor imaging study. Alcohol Clin Exp Res 36, 1922-1931.

[129] Foley JM, Salat DH, Stricker NH, Zink TA, Grande LJ, McGlinchey RE, Milberg WP, Leritz EC (2014) Interactive effects of apolipoprotein $\mathrm{E} 4$ and diabetes risk on later myelinating white matter regions in neurologically healthy older aged adults. Am J Alzheimers Dis Other Demen 29, 222-235.

[130] Stricker NH, Salat DH, Kuhn TP, Foley JM, Price JS, Westlye LT, Esterman MS, McGlinchey RE, Milberg WP, Leritz EC (2016) Mild Cognitive Impairment is Associated With White Matter Integrity Changes in LateMyelinating Regions Within the Corpus Callosum. Am J Alzheimers Dis Other Demen 31, 68-75.

[131] Stricker NH, Salat DH, Foley JM, Zink TA, Kellison IL, McFarland CP, Grande LJ, McGlinchey RE, Milberg WP, Leritz EC (2013) Decreased white matter integrity in neuropsychologically defined mild cognitive impairment is independent of cortical thinning. J Int Neuropsychol Soc 19, 925-937.

[132] Stricker NH, Schweinsburg BC, Delano-Wood L, Wierenga CE, Bangen KJ, Haaland KY, Frank LR, Salmon DP, Bondi MW (2009) Decreased white matter integrity in late-myelinating fiber pathways in Alzheimer's disease supports retrogenesis. Neuroimage 45, 10-16.

[133] Rose SE, Chen F, Chalk JB, Zelaya FO, Strugnell WE, Benson M, Semple J, Doddrell DM (2000) Loss of connectivity in Alzheimer's disease: an evaluation of white matter tract integrity with colour coded MR diffusion tensor imaging. J Neurol Neurosurg Psychiatry 69, 528-530.

[134] Kaskikallio A, Karrasch M, Rinne JO, Tuokkola T, Parkkola R, Gronholm-Nyman P (2019) Domain-specific cognitive effects of white matter pathology in old age, mild cognitive impairment and Alzheimer's disease. Neuropsychol Dev Cogn B Aging Neuropsychol Cogn, 1-18.

[135] Kaskikallio A, Karrasch M, Rinne JO, Tuokkola T, Parkkola R, Gronholm-Nyman P (2019) Domain-specific cognitive effects of white matter pathology in old age, mild cognitive impairment and Alzheimer's disease. Neuropsychol Dev Cogn B Aging Neuropsychol Cogn, 1-18.

[136] Tullberg M, Fletcher E, DeCarli C, Mungas D, Reed BR, Harvey DJ, Weiner MW, Chui HC, Jagust WJ (2004) White matter lesions impair frontal lobe function regardless of their location. Neurology 63, 246-253.

[137] Li X, Westman E, Stahlbom AK, Thordardottir S, Almkvist O, Blennow K, Wahlund LO, Graff C (2015) White matter changes in familial Alzheimer's disease. J Intern Med 278, 211-218.

[138] Lee S, Viqar F, Zimmerman ME, Narkhede A, Tosto G, Benzinger TL, Marcus DS, Fagan AM, Goate A, Fox NC, Cairns NJ, Holtzman DM, Buckles V, Ghetti B, McDade E, Martins RN, Saykin AJ, Masters CL, Ringman JM, Ryan NS, Forster S, Laske C, Schofield PR, Sperling RA, Salloway S, Correia S, Jack C, Jr., Weiner M, Bateman RJ, Morris JC, Mayeux R, Brickman AM (2016) White matter hyperintensities are a core feature of Alzheimer's disease: Evidence from the dominantly inherited Alzheimer network. Ann Neurol 79, 929939.

[139] Roher AE, Maarouf CL, Malek-Ahmadi M, Wilson J, Kokjohn TA, Daugs ID, Whiteside CM, Kalback WM, Macias MP, Jacobson SA, Sabbagh MN, Ghetti B, Beach TG (2013) Subjects harboring presenilin familial Alzheimer's disease mutations exhibit diverse white matter biochemistry alterations. Am J Neurodegener Dis 2, 187-207. 
[140] Tanskanen M, Makela M, Myllykangas L, Rastas S, Sulkava R, Paetau A (2012) Intracerebral hemorrhage in the oldest old: a population-based study (vantaa 85+). Front Neurol 3, 103-

[141] Kantarci K, Gunter JL, Tosakulwong N, Weigand SD, Senjem MS, Petersen RC, Aisen PS, Jagust WJ, Weiner MW, Jack CR, Jr. (2013) Focal hemosiderin deposits and beta-amyloid load in the ADNI cohort. Alzheimers Dement 9, S116-S123.

[142] Wu A, Sharrett AR, Gottesman RF, Power MC, Mosley TH, Jr., Jack CR, Jr., Knopman DS, Windham BG, Gross AL, Coresh J (2019) Association of Brain Magnetic Resonance Imaging Signs With Cognitive Outcomes in Persons With Nonimpaired Cognition and Mild Cognitive Impairment. JAMA Netw Open 2, e193359-

[143] Zamrini E, Adler C, Serrano G, Zhang N, Driver-Dunckley E, Mehta S, Shprecher D, Sabbagh M, Shill H, Sue L, Beach T (2019) Clinicopathological correlates of aging-related tau astrogliopathy (ARTAG). International Congress of Parkinson's Disease and Movement Disorders

[144] Ding ZT, Wang Y, Jiang YP, Yoshida M, Mimuro M, Inagaki T, Iwase T, Hashizume Y (2006) Argyrophilic grain disease: frequency and neuropathology in centenarians. Acta Neuropathol 111, 320-328.

[145] Rodriguez RD , Grinberg LT (2015) Argyrophilic grain disease: An underestimated tauopathy. Dement Neuropsychol 9, 2-8.

[146] Yokota O, Miki T, Ikeda C, Nagao S, Takenoshita S, Ishizu H, Haraguchi T, Kuroda S, Terada S, Yamada N (2018) Neuropathological comorbidity associated with argyrophilic grain disease. Neuropathology 38, 82-97.

[147] Robinson JL, Corrada MM, Kovacs GG, Dominique M, Caswell C, Xie SX, Lee VM, Kawas CH, Trojanowski JQ (2018) Non-Alzheimer's contributions to dementia and cognitive resilience in The 90+ Study. Acta Neuropathol 136, 377-388.

[148] Liu AK, Goldfinger MH, Questari HE, Pearce RK, Gentleman SM (2016) ARTAG in the basal forebrain: widening the constellation of astrocytic tau pathology. Acta Neuropathol Commun 4, 59-

[149] Kovacs GG, Lee VM, Trojanowski JQ (2017) Protein astrogliopathies in human neurodegenerative diseases and aging. Brain Pathol 27, 675-690.

[150] Sabbagh MN, Sandhu SS, Farlow MR, Vedders L, Shill HA, Caviness JN, Connor DJ, Sue L, Adler CH, Beach TG (2009) Correlation of clinical features with argyrophilic grains at autopsy. Alzheimer Dis Assoc Disord 23, 229-233.

[151] Hoglinger GU, Respondek G, Stamelou M, Kurz C, Josephs KA, Lang AE, Mollenhauer B, Muller U, Nilsson C, Whitwell JL, Arzberger T, Englund E, Gelpi E, Giese A, Irwin DJ, Meissner WG, Pantelyat A, Rajput A, van Swieten JC, Troakes C, Antonini A, Bhatia KP, Bordelon Y, Compta Y, Corvol JC, Colosimo C, Dickson DW, Dodel R, Ferguson L, Grossman M, Kassubek J, Krismer F, Levin J, Lorenzl S, Morris HR, Nestor P, Oertel WH, Poewe W, Rabinovici G, Rowe JB, Schellenberg GD, Seppi K, van ET, Wenning GK, Boxer AL, Golbe LI, Litvan I (2017) Clinical diagnosis of progressive supranuclear palsy: The movement disorder society criteria. Mov Disord 32, 853-864.

[152] Respondek G, Roeber S, Kretzschmar H, Troakes C, Al-Sarraj S, Gelpi E, Gaig C, Chiu WZ, van Swieten JC, Oertel WH, Hoglinger GU (2013) Accuracy of the National Institute for Neurological Disorders and Stroke/Society for Progressive Supranuclear Palsy and neuroprotection and natural history in Parkinson plus syndromes criteria for the diagnosis of progressive supranuclear palsy. Mov Disord 28, 504-509. 
[153] Sakamoto R, Tsuchiya K, Mimura M (2010) Clinical heterogeneity in progressive supranuclear palsy: problems of clinical diagnostic criteria of NINDS-SPSP in a retrospective study of seven Japanese autopsy cases. Neuropathology 30, 24-35.

[154] Respondek G, Stamelou M, Kurz C, Ferguson LW, Rajput A, Chiu WZ, van Swieten JC, Troakes C, Al SS, Gelpi E, Gaig C, Tolosa E, Oertel WH, Giese A, Roeber S, Arzberger T, Wagenpfeil S, Hoglinger GU (2014) The phenotypic spectrum of progressive supranuclear palsy: a retrospective multicenter study of 100 definite cases. Mov Disord 29, 1758-1766.

[155] Yoshida K, Hata Y, Kinoshita K, Takashima S, Tanaka K, Nishida N (2017) Incipient progressive supranuclear palsy is more common than expected and may comprise clinicopathological subtypes: a forensic autopsy series. Acta Neuropathol 133, 809-823.

[156] Beach TG , Adler CH (2018) Importance of low diagnostic Accuracy for early Parkinson's disease. Mov Disord 33, 1551-1554.

[157] Nogami A, Yamazaki M, Saito Y, Hatsuta H, Sakiyama Y, Takao M, Kimura K, Murayama S (2015) Early Stage of Progressive Supranuclear Palsy: A Neuropathological Study of 324 Consecutive Autopsy Cases. J Nippon Med Sch 82, 266-273.

[158] Respondek G, Kurz C, Arzberger T, Compta Y, Englund E, Ferguson LW, Gelpi E, Giese A, Irwin DJ, Meissner WG, Nilsson C, Pantelyat A, Rajput A, van Swieten JC, Troakes C, Josephs KA, Lang AE, Mollenhauer B, Muller U, Whitwell JL, Antonini A, Bhatia KP, Bordelon Y, Corvol JC, Colosimo C, Dodel R, Grossman M, Kassubek J, Krismer F, Levin J, Lorenzl S, Morris H, Nestor P, Oertel WH, Rabinovici GD, Rowe JB, van ET, Wenning GK, Boxer A, Golbe LI, Litvan I, Stamelou M, Hoglinger GU (2017) Which ante mortem clinical features predict progressive supranuclear palsy pathology? Mov Disord 32, 995-1005. 